\title{
Score Based Network Selection in Heterogeneous Wireless Networks to Enhance Quality of Service for End User
}

\author{
Piyush K. Ingole ${ }^{1}$, M. V. Sarode ${ }^{2}$ and Meenakshi S. Arya ${ }^{3}$ \\ ${ }^{1}$ Research Scholar, ${ }^{2}$ Supervisor, ${ }^{3}$ Head, CSE \\ ${ }^{1,2 \& 3}$ G.H. Raisoni College of Engineering, Maharashtra, India \\ E-Mail: piyush.ingole@gmail.com
}

\begin{abstract}
Abrupt node movements is mobile networks directly affects the network connectivity of the node. Abruptly moving nodes generally face the issue of low network quality, and call drops. An effective handoff mechanism is needed in order to address this issue. In this research, we are proposing a network score based handoff mechanism, which follows the makebefore-break principle. An improvement in terms of handoff delay, and packet loss is reported by the extensive analysis and simulations performed on the proposed system. Average handoff delay of less than $1 \mathrm{~ms}$, and packet loss ratio of less than $1 \%$ are obtained by our research. We plan to extend the research further for multiple networks to extend the performance analysis
\end{abstract}

Keywords: Handoff, Make before Break, Score Based, Delay, Packet Loss Ratio

\section{INTRODUCTION}

Effective handoff in wireless networks has been a major research area since the introduction of voice calls. Researchers have proposed various techniques for handoff, including but not limited to single parameter handoffs, multi parameter handoffs, make before break, break before make, fuzzy logic based handoffs, and many more. These handoff techniques usually consider the network state, the node state and the network structure for taking handoff decisions. While, some of these techniques are superior than others, the multi parameter handoff technique based on make before break, performs better under practical network conditions.

Score based handoff mechanisms is the preferred choice by many network providers, mainly due to it's flexibility to add multiple parameters to the handoff evaluation process, and extension of the process to multiple heterogeneous networks. Using the score based handoff mechanism, the overall handoff performance can be controlled by tuning the parameters and the network conditions used in handoff.

Handoff decisions are taken either by a hard controlled or soft controlled system. Hard controlled systems use a Yes/No mechanism for scoring the handoff decision per parameter. The Yes/No scores are then aggregated to form a final score. This final score is then given to a threshold device in order to take the handoff decision. The hard controlled systems are generally effective for handing off between 2 networks, but as number of networks increase, the complexity of handoff increases. This reduces the handoff efficiency of the hard controlled systems.

Soft controlled systems are based on Fuzzy decisions or bioinspired decisions. These systems can be scaled over multiple heterogeneous networks, over multiple parameters. Usually soft controlled systems first fuzzify the input parameters into ranges, and then apply decision criteria on these inputs. These criterion are based on the required network and node performance. Usually, for delay tolerant networks, fast handoffs are needed, for which the soft controlled systems are tuned to have minimal primary parameters, and minimal decision complexity. Whereas, for high performance networks, the handoff parameters are decided for highest network connectivity, and better network connection.

Practical wireless networks are usually placed between delay tolerant networks and high performance networks, so, the handoff criterion for these networks is also selected to be in between delay improvement and overall network performance improvement. Our approach targets this class of practical general purpose wireless networks, by combining the optimizations from delay tolerant networks and high performance networks. We simulated our network under various conditions, and checked the performance of the system in terms of handoff delay and packet loss due to handoff, and optimum results were obtained. In our simulations, variations in terms of network size, node density, node speed and node communication were performed to test the viability of the technique for practical network scenarios. Handoff delay usually varies between $100 \mathrm{~ns}$ to $1 \mathrm{~ms}$ under various test conditions, while the packet loss ratio usually varies between $0.1 \%$ to $1 \%$, thereby giving a very optimum structure for real time network communications.

\section{LITERATURE REVIEW}

Handoff is a mainstream term utilized as a part of cellular communication framework. Handoff is alluded to as the way of exchanging a call starting with one base station then onto the next without disengaging it. This component enhances the portability of a communication framework given to the client. Handoff, happens when the client is moving and the signal quality of the following base station 
is more grounded than the signal quality of the base station to which mobile unit is at presently associated. Quantities of handoff methodologies have been clarified by analysts and approaches to enhance portability which are additionally been presented beneath.

AbhinavKumar et al., (2013) has stamped portability as an essential factor that chooses the execution and nature of any communication framework. Handoff is essential for congruity of call which is one of QoS parameters that chooses the nature of thenetwork. In this paper, the authors have quickly portrayed different systems of handoff and have made acorrelation between various handoff techniques that are utilized as a part of remote cell communication. The examination is made on the premise of different parameters like execution time, S/I proportion, Relative Signal quality, call taking care of trouble, handoff made and age strategies.

Nisha et al., (2013) expresses that handoff between various cells are unavoidable and it is imperative to keep away from call separation. Quantities of investigates have been done to consider unsuccessful handoffs. The authors have completed research to consider the effective channel assignment and have likewise contemplated diverse techniques of handoff to discover QoS parameters to assess the quality given to portable clients. The strategies of handover have been concluded on the premise of hysteresis, signal quality, and limit. Rundown of various handoff methodologies is likewise given in the paper. In the paper, discourse is done on different handoff conventions, handoff administration issues and on handoff choices.

Vidya S. Pande (2013) has specified the advancing technology as a purpose behind quick advances in wireless communication frameworks. Advances are being made in the administrations gave to the clients and the quality is being enhanced with the goal that clients can utilize this innovation whenever and anyplace. In this paper proposition of handoff calculation amongst WLAN and CDMA, 2000 cell networks are given. The handoff in the proposed calculation is done on the premise of the handoff delay and throughput. A perfect vertical handoff method between IEEE 802.11 WLAN is proposed in the paper.

Madan Lal Tetarwal et al., (2014) expresses the upsides of wireless communication frameworks, for example, adaptability, portability, and versatility. Heaps of inquires have been done to diminish delays caused by handoffs. Author expresses that Handoff is more imperative in WLAN when contrasted with communication frameworks in view of therestricted scope of APs in WLAN. Authors have proposed different plans for lessening handoff dormancy and to empower fast handoff in IEEE 802.11 remote networks. An audit of different quick handoff systems have been done and after that are recorded its benefits and bad marks. The motivation behind the author of the paper is to decrease handoff inertness for ITS in vehicles and to stamp the significance of quick handoff for theimpeccable network. Authors have attempted to decrease handoff delays by proposing different strategies.

Piyush S. Jirapure et al., (2014) have said portability as a vital figure contributing assessing the execution of remote networks. Portability is just conceivable because of handoff. The administration progression entirely relies upon the handoff that happens while thecall is to be exchanged starting with one cell then onto the next. A few choices are to be made while exchanging a call i.e. choice of best network is done as such that call proceeds. Handoff choice systems are proposed in the paper that deals with the premise of specific parameters like RSS Based, Cost work Based, QoS Based, Processing Delay Based, Policy-Based, Context-Aware Based and so on. The execution criteria of the proposed techniques are measured and handoff parameters are computed.

Geetanjali Chellani et al., (2013) have talked about different handover systems by characterizing and looking at them. The customary procedures of handoff have been talked about in the paper that was acquainted with improve the execution of remote communication frameworks amid handoff. Arrangements are given in the paper to defeat the issues looked in the traditional frameworks and a short exchange is done on the proposed procedures.

\section{PROPOSED HANDOFF TECHNIQUE}

Our proposed score based soft handoff technique is shown in Figure 1. Initially, the network parameters like node RSSI, SNR, bandwidth and data rate are sensed. These parameters are then given to a selection engine, which selects the best parameters for handoff decision. These parameters are selected based on the requirement of the network designer. If the network designer needs handoff only on the basis of RSSI and data rate, then the selection unit is tuned accordingly to produce only RSSI and data rate at the output. In our case, we are using all the 4 parameters for handoff decision.

These selected parameters are then given to a fuzzification block, where the parameters are converted into ranges of low, medium and high for application of handoff rules. The rules are in the form of if-else conditions, which convert the sensed parameters into scores between the two networks. For example, if the bandwidth of a node provided by network 1 is more than that provided by network 2 , then the score of network 1 is increased by a weighted constant value.

This weighted constant value is setup according to the necessity of a particular parameter under the given network conditions. In case, the network designer needs that SNR should be of higher importance than RSSI, then weighted constant for SNR will be higher than the weighted constant of RSSI. 


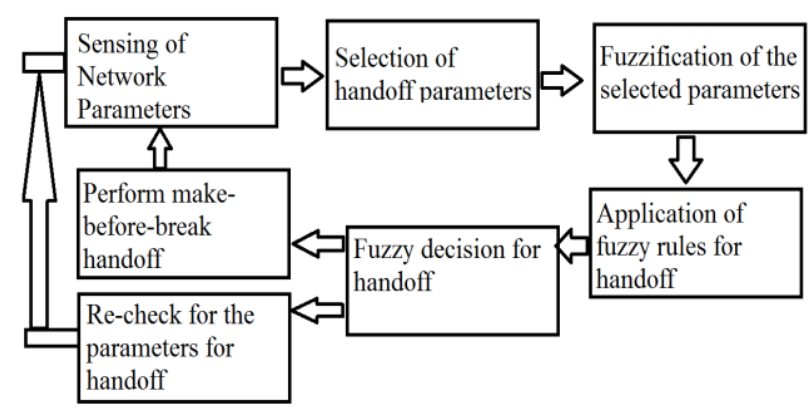

Fig. 1 Proposed Handoff Technique

The scores for both the networks are evaluated, and are then given to a fuzzy decision engine. This engine compares these scores, and then decides whether or not handoff should take place. If the score of network 1 is higher than that of network 2, and the node is currently in network 2, then the node is handed over from network 2 to network 1 by using a make-before-break handoff procedure.

But, if the score of network 1 is more than that of network 2 , and the node is in network 1 , then there is no need of handoff, and the system re-scans the parameters of the nodes. This process goes on for the entire network lifetime, and handoffs are done based on the described fuzzy decisions. Analysis of the system is explained in section 4, and it shows that the overall network handoff performance is optimum.

\section{PERFORMANCE ANALYSIS}

We are using network simulator ver 2.29 for performance analysis of the developed handoff system. The Multi Handoff Criteria (MIH) patch has been used while developing the system, and has been integrated with the ns2.29 installation. Table 1 indicates the network parameters of the system.

TABLE I NETWORK PARAMETERS

\begin{tabular}{|l|c|}
\hline \multicolumn{1}{|c|}{ Parameter Name } & Value \\
\hline MAC Layer & 802.21 \\
\hline Network Size & 100x100 to 500x500 \\
\hline Number of nodes & 20 to 100 \\
\hline Node mobility & Upto $100 \mathrm{kmph}$ \\
\hline Number of communications & 10 to 100 \\
\hline Number of networks & 2 (WiFi and WiMAX) \\
\hline
\end{tabular}

Once the network parameters are setup, then we tested the system based on specific ranges of RSSI, SNR, Data rate and bandwidth for the WiFi and WiMAX networks. We then changed the number of nodes, did some variations in the number of communications, and then analyzed the effect of our algorithm on average handoff delay, and the packet loss ratio. Table II depicts the changes in these parameters over node quantity variations, Table III depicts the changes in these parameters over changes in mobility variation, Table IV shows the variations in the parameters over network size variations, and finally Table $\mathrm{V}$ shows the variations in delay and packet loss over varying number of communications.

TABle I I Number Of Nodes V/S Handoff Delay \& PlR

\begin{tabular}{|c|c|c|}
\hline $\begin{array}{c}\text { Number of } \\
\text { Nodes }\end{array}$ & $\begin{array}{c}\text { Average Handoff } \\
\text { Delay (ms) }\end{array}$ & $\begin{array}{c}\text { Packet Loss } \\
\text { Ratio (\%) }\end{array}$ \\
\hline 10 & 0.4 & 0.1 \\
\hline 20 & 0.45 & 0.12 \\
\hline 30 & 0.52 & 0.18 \\
\hline 50 & 0.69 & 0.35 \\
\hline 75 & 0.74 & 0.38 \\
\hline 100 & 0.76 & 0.39 \\
\hline
\end{tabular}

As depicted by table II, the handoff delay and packet loss ratio increases linearly for less number of nodes, but becomes almost saturated around the range of $0.7 \mathrm{~ms}$ for handoff delay and $0.38 \%$ for packet loss ratio.

TABle III Node Mobility V/S HANdoff DelAy \& PlR

\begin{tabular}{|c|c|c|}
\hline $\begin{array}{c}\text { Node Mobility (kmph) } \\
(\mathbf{N N}=\mathbf{1 0 0})\end{array}$ & $\begin{array}{c}\text { Average Handoff } \\
\text { Delay (ms) }\end{array}$ & $\begin{array}{c}\text { Packet Loss } \\
\text { Ratio (\%) }\end{array}$ \\
\hline 0 & 0.65 & 0.2 \\
\hline 5 & 0.71 & 0.35 \\
\hline 20 & 0.74 & 0.38 \\
\hline 40 & 0.75 & 0.57 \\
\hline 80 & 0.78 & 0.72 \\
\hline 100 & 0.79 & 0.82 \\
\hline
\end{tabular}

Table III shows that the packet loss ratio of the network increases exponentially as the node mobility increases, this is due to the fact that node speed has a big impact on the number of packets dropped by the node. While the network delay remains almost constant for constant number of nodes

TABle Iv Network Size V/S HANDoff Delay \& PlR

\begin{tabular}{|c|c|c|}
\hline $\begin{array}{c}\text { Network size } \\
(\mathbf{X} * \mathbf{Y}) \\
(\mathbf{N N}=\mathbf{1 0 0})\end{array}$ & $\begin{array}{c}\text { Average Handoff } \\
\text { Delay }(\mathbf{m s})\end{array}$ & $\begin{array}{c}\text { Packet Loss } \\
\text { Ratio }(\%)\end{array}$ \\
\hline $100 \times 100$ & 0.52 & 0.53 \\
\hline $200 \times 200$ & 0.59 & 0.61 \\
\hline $300 \times 300$ & 0.63 & 0.68 \\
\hline $400 \times 400$ & 0.71 & 0.72 \\
\hline $450 \times 450$ & 0.74 & 0.75 \\
\hline $500 \times 500$ & 0.79 & 0.78 \\
\hline
\end{tabular}

Table IV shows that the packet loss ratio and the average handoff delay of the network increases linearly as the network size increases. This is expected, as due to increase in the network area, the distance between the nodes also increases, and thus it increases the delay of signal transmission, and due to increase in the distance, the packet delivery of the nodes also reduces, thereby increasing the packet loss ratio. 
TABle V Number Of COMmunications V/S HANdoff Delay \& PlR

\begin{tabular}{|c|c|c|}
\hline $\begin{array}{c}\text { Number of comm } \\
(\mathbf{N N}=\mathbf{1 0 0})\end{array}$ & $\begin{array}{c}\text { Average Handoff } \\
\text { Delay (ms) }\end{array}$ & $\begin{array}{c}\text { Packet Loss } \\
\text { Ratio (\%) }\end{array}$ \\
\hline 10 & 0.72 & 0.24 \\
\hline 20 & 0.725 & 0.31 \\
\hline 40 & 0.728 & 0.45 \\
\hline 60 & 0.73 & 0.59 \\
\hline 80 & 0.734 & 0.63 \\
\hline 100 & 0.741 & 0.81 \\
\hline
\end{tabular}

Table $\mathrm{V}$ shows that the packet loss ratio of the network increases linearly with number of communications, while the average handoff delay is almost constant.

The handoff delay under the given variation of simulation conditions can be plotted as shown in figure 2. While the packet loss ratio (PLR) is shown in figure 3.

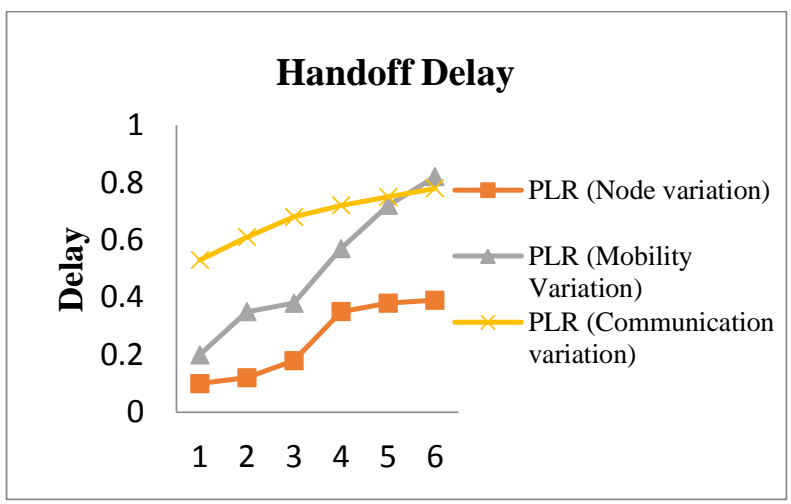

Fig. 2 Handoff delay under different parameter variations

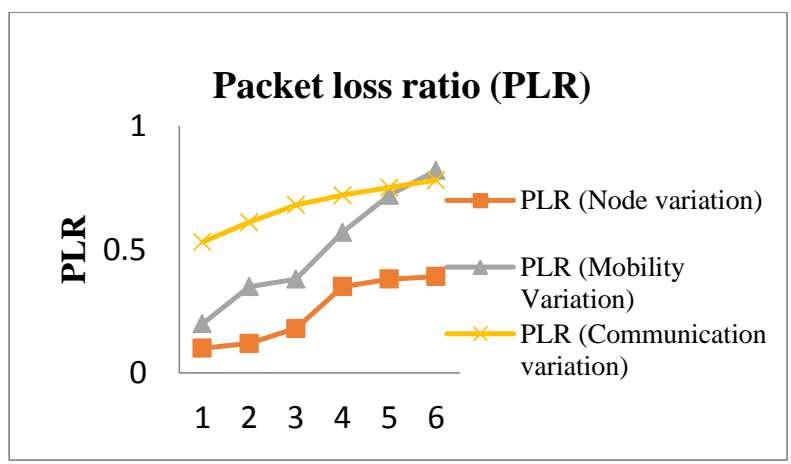

Fig. 3 Variation of PLR under different parameter variations
Both the graphs indicate the min and max ranges for the simulation outputs. The handoff delay varies from $0.4 \mathrm{~ms}$ to $0.79 \mathrm{~ms}$, which is very low when compared to conventional methods, while the packet loss rate is maintained below $1 \%$ for the entire handoff process.

\section{CONCLUSION}

Analysis of the score based handoff mechanism indicates that the average handoff delay and the packet loss ratio of the network is very low. This indicates that the proposed algorithm can be used for various practical network scenarios like VANETs, MANETs and other mobile networks. We would be extending our research by adding multiple heterogeneous networks, and also by increasing the number of parameters for handoff decision. Further analysis can be done by implementing the handoff procedure in real time, and observing the change in the network performance under a physical network condition.

\section{REFERENCES}

[1] Abhinavkumar and Hemant Purohit, "A Comparative Study of Different Types of Handoff Strategies in Cellular Systems", IJARCCE, Vol. 2, No. 11, pp. 4278-4287, November 2013.

[2] Nisha, Sunil Kumar and Jyoti Bhatnagar, "Handoff Strategies in Cellular System", IJNTEC, Vol. 1, No. 2, pp. 22-28, August, 2013.

[3] Vidya S. Pande, "Review Paper on Vertical Handoff Algorithm between IEEE 802.11 WLAN \& CDMA Cellular Network", IJECCE, Vol. 4, No. 2, pp. 40-42, 2013.

[4] Madan Lal Tetarwal, "A Review on Handoff Latency Reducing Techniques in IEEE 802.11 WLAN", IJCA, pp. 22-28, 2014.

[5] Piyush S. Jirapure, Amarsinh V. Vidhate, "Survey and Analysis of Handoff Decision Strategies for Heterogeneous Mobile Wireless Networks", IJARCSSE, Vol. 4, No. 4, pp. 703-713, April 2014.

[6] Geetanjali Chellani, Anshuman Kalla "A Review: Study of Handover Performance in Mobile IP", IJCNC, Vol. 5, No. 6, pp. 137-151, November 2013. 